\title{
A Case Report of Subcutaneous Insulin Resistance in Type 1 Diabetes, July 2018, Ardabil, Iran
}

\section{Zamanpour Mohammadzaman ${ }^{1}$, Maskani Reza ${ }^{1 *}$ and Hosseini Anbaran Sonia ${ }^{2}$}

${ }^{1}$ Pediatric Department, Buali Children's Hospital, Ardabil University of Medical Sciences (ARUMS), Iran

${ }^{2}$ Department of Medical, Ardabil Branch, Ardabil University of Medical Sciences (ARUMS), Iran

*Corresponding Author: Maskani Reza, Pediatric Department, Buali Children's Hospital, Ardabil University of Medical Sciences (ARUMS), Iran.

Received: August 07, 2019; Published: September 12, 2019

DOI: 10.31080/ASPE.2019.02.0146

\begin{abstract}
Extreme subcutaneous insulin resistance is a rare syndrome characterized by severe resistance to subcutaneous insulin with normal intravenous insulin sensitivity. It is pathophysiology is unknown, though an increased insulin degrading activity has been suggested [1]. We report a case of extreme insulin resistance in a stable, diabetic patient without any acute complication related to diabetes mellitus. A 6 year old diabetic patient, from Ardabil, that presented with unusually uncontrolled diabetes mellitus due to extreme insulin resistance. That finally our patient was treated by Lantus, Apidra and Rituximab.
\end{abstract}

Keywords: Diabetes Mellitus; Insulin Resistance; Subcutaneous Injection

\section{Introduction}

Hyperglycemia is common in hospitalized patients. A lot of factors including underlying medical conditions, stress and some medications can contribute to transient hyperglycemia in diabetic as well as non-diabetic patients. But persistent hyperglycemia for a long period in spite of management following guidelines is usually associated with an underlying pathology. Insulin resistance is a known cause of hyperglycemia. On a clinical basis, severe insulin resistance is defined as a situation in which a patient requires more than 200 units of insulin daily for more than 2 days [2,6]. Though physicians are familiar with common diseases that are known to be associated with insulin resistance, the majority of us rarely come across a case of extreme insulin resistance. It is usually seen during the attack of diabetic ketoacidosis, although non-ketoacidosis patients can also develop sever insulin resistance [6].

\section{Case Presentation}

We report a case of extreme insulin resistance in a stable, diabetic patient without any acute complication related to diabetes mellitus. Our patient was a 6-year old male that had diabetes 3 years ago. He was from Ardabil, Iran. He presented with unusually uncontrolled diabetes mellitus due to extreme insulin resistance. Despite dramatic increase in insulin step by step up to 80 IU of concomitant short and intermediate acting insulin per day by subcutaneous route (his BW:20 kg). The patient was not controlled with low doses of subcutaneous insulin. However, it controlled it with low doses of intravenous insulin, but it suffered from a reduction in blood glucose, which continued despite the loss of intravenous insulin. Glucose in the patient's blood again increased. So, we injected a dose of IVIG into the patient and changed the patient's insulin from NPH and Regular to Lantus and Apidra (more than 3 $\mathrm{IU} / \mathrm{kg}$ in day). Finally, the patient's blood glucose remained at normal until 9 months. Insulin requirements rose again and we had to use Rituximab, which also reduced the need for insulin after using Rituximab. His blood test for insulin autoantibody was negative. However, we could not measure insulin receptor antibodies and we do not believe they played a role because of the absence of clinical 
features of an autoimmune disease or malignancy in this case. Insulin, plasma leptin and serum levels could not be measured due to unavailability of these tests in our country. With these limitations, we could not explore the exact mechanism for the extreme insulin resistance in this case.

\section{Discussion}

Insulin resistance has been defined as "a state (of a cell, tissue or organism) in which a greater than normal amount of insulin is required to elicit a quantitatively normal response". Severe insulin resistance should be suspected when an individual requires more than 2 units $/ \mathrm{kg} /$ day of insulin. Extreme subcutaneous insulin resistance is characterized by severe resistance to subcutaneous insulin with normal or near normal intravenous insulin sensitivity. Established mechanisms of insulin resistance include genetic defects in insulin receptors, insulin receptor antibodies and interference with intracellular insulin action due to the excess of counter-regulatory hormones of inflammatory cytokines and increased insulin clearance [6].

We have described a case of severe subcutaneous insulin resistance that was treated by Rituximab. Such cases have already been described. For some of them, an insulin-degrading activity has been found in muscle or adipose tissue. Therefore, some patients were successfully treated with a protease inhibitor, aprotinin. Other patients did not respond to this treatment [3]. Intramuscular insulin therapy was successfully attempted in some cases [4,5]. All of these treatments were ineffective in our patient. Intraperitoneal insulin therapy, through a subcutaneous access device, was proposed in some cases with good results [3].

\section{Conclusion}

Extreme subcutaneous insulin resistance is a rare syndrome characterized by severe resistance to subcutaneous insulin with normal intravenous insulin sensitivity. It is pathophysiology is unknown, though an increased insulin degrading activity has been suggested. Management of such cases is more challenging [1]. Your patient was treated by Lantus, Apidra and Rituximab.

\section{Bibliography}

1. Soudan B., et al. "Extreme subcutaneous insulin resistance: a misunderstood syndrome". Diabetes and Metabolism 29.5 (2003): 539-546.
2. Ballani P., et al. "Clinical experience with U-500 regular insulin in obese, markedly insulin resistant Type-2 diabetic patients". Diabetes Care 29.11 (2006): 2504-2505.

3. Riveline JP., et al. "Extreme subcutaneous insulin resistance successfully treated by an implantable pump". Diabetes Care. 24.12 (2001): 2155-2156.

4. Brossard JH., et al. "Long-term use of intramuscular insulin therapy in a type I diabetic patient with subcutaneous insulin resistance". Diabetic Medicine 10.2 (1993): 174-176.

5. Pickup JC., et al. "Management of severely brittle diabetes by continuous subcutaneous and intramuscular insulin infusions: evidence for a defect in subcutaneous insulin absorption". The British Medical Journal 282.6261 (1981): 347-350.

6. Zuhayer Ahmed., et al. "A Male with Extreme Subcutaneous Insulin Resistance: A Case Report". Romanian Journal of Diabetes Nutrition and Metabolic Diseases 23.2 (2016): 209-213.

Volume 2 Issue 10 October 2019 (C) All rights are reserved by Maskani Reza., et al. 